\title{
Statistical Analysis Techniques in Particle Physics
}

Fits, Density Estimation and Supervised Learning

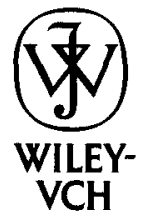




\section{Contents}

Acknowledgements XIII

\section{Notation and Vocabulary $X V$}

1 Why We Wrote This Book and How You Should Read It 1

2 Parametric Likelihood Fits 5

2.1 Preliminaries 5

2.1.1 Example: CP Violation via Mixing 7

2.1.2 The Exponential Family 9

2.1.3 Confidence Intervals 10

2.1.4 Hypothesis Tests 11

2.2 Parametric Likelihood Fits 12

2.2.1 Nuisance Parameters 16

2.2.2 Confidence Intervals from Pivotal Quantities 17

2.2.3 Asymptotic Inference 19

2.2.4 Profile Likelihood 20

2.2.5 Conditional Likelihood 20

2.3 Fits for Small Statistics 21

2.3.1 Sample Study of Coverage at Small Statistics 22

2.3.2 When the pdf Goes Negative 25

2.4 Results Near the Boundary of a Physical Region 26

2.5 Likelihood Ratio Test for Presence of Signal 28

$2.6 \quad \%$ sPlots 31

$2.7 \quad$ Exercises 35

References 37

3 Goodness of Fit 39

3.1 Binned Goodness of Fit Tests 41

3.2 Statistics Converging to Chi-Square 46

3.3 Univariate Unbinned Goodness of Fit Tests 49

3.3.1 Kolmogorov-Smirnov 49

3.3.2 Anderson-Darling 50

3.3.3 Watson 51

3.3.4 Neyman Smooth 51 
VI Contents

3.4 Multivariate Tests 52

3.4.1 Energy Tests 53

3.4.2 Transformations to a Uniform Distribution 54

3.4.3 Local Density Tests 55

3.4.4 Kernel-based Tests 56

3.4.5 Mixed Sample Tests 57

3.4.6 Using a Classifier 58

$3.5 \quad$ Exercises 59

References 61

$4 \quad$ Resampling Techniques 63

4.1 Permutation Sampling 63

4.2 Bootstrap 65

4.2.1 Bootstrap Confidence Intervals 68

4.2.2 Smoothed Bootstrap 70

4.2.3 Parametric Bootstrap 70

$4.3 \quad$ Jackknife 70

4.4 $\quad \mathrm{BC}_{\mathrm{a}}$ Confidence Intervals 76

4.5 Cross-Validation 78

$4.6 \quad 82$

4.7 Exercises 86

References 86

5 Density Estimation 89

5.1 Empirical Density Estimate 90

5.2 Histograms 90

5.3 Kernel Estimation 92

5.3.1 Multivariate Kernel Estimation 92

5.4 Ideogram 93

5.5 Parametric vs. Nonparametric Density Estimation 93

5.6 Optimization 94

5.6.1 Choosing Histogram Binning 97

5.7 Estimating Errors 100

5.8 The Curse of Dimensionality 102

5.9 Adaptive Kernel Estimation 103

$5.10 \quad$ Naive Bayes Classification 105

5.11 Multivariate Kernel Estimation 106

5.12 Estimation Using Orthogonal Series 108

5.13 Using Monte Carlo Models 111

$5.14 \quad$ Unfolding 112

5.14.1 Unfolding: Regularization 116

5.15 Exercises 120

References 120

6 Basic Concepts and Definitions of Machine Learning 121

6.1 Supervised, Unsupervised, and Semi-Supervised 121 
6.2 Tall and Wide Data 123

6.3 Batch and Online Leaming 124

6.4 Parallel Learning 125

6.5 Classification and Regression 127

References 128

7 Data Preprocessing 129

7.1 Categorical Variables 129

7.2 Missing Values 132

7.2.1 Likelihood Optimization 134

7.2.2 Deletion 135

7.2.3 Augmentation 137

7.2.4 Imputation 137

7.2.5 Other Methods 139

7.3 Outliers 139

7.4 Exercises 141

References 142

8 Linear Transformations and Dimensionality Reduction 145

8.1 Centering, Scaling, Reflection and Rotation 145

8.2 Rotation and Dimensionality Reduction 146

8.3 Principal Component Analysis (PCA) 147

8.3.1 Theory 148

8.3.2 Numerical Implementation 149

8.3.3 Weighted Data 150

8.3.4 How Many Principal Components Are Enough? 151

8.3.5 Example: Apply PCA and Choose the Optimal Number of Components 154

8.4 Independent Component Analysis (ICA) 158

8.4.1 Theory 158

8.4.2 Numerical implementation 161

8.4.3 Properties 162

8.5 Exercises 163

References 163

9 Introduction to Classification 165

9.1 Loss Functions: Hard Labels and Soft Scores 165

9.2 Bias, Variance, and Noise 168

9.3 Training, Validating and Testing: The Optimal Splitting Rule 173

9.4 Resampling Techniques: Cross-Validation and Bootstrap 177

9.4.1 Cross-Validation 177

9.4.2 Bootstrap 179

9.4.3 Sampling with Stratification 181

9.5 Data with Unbalanced Classes 182

9.5.1 Adjusting Prior Probabilities 183

9.5.2 Undersampling the Majority Class 184 
9.5.3 Oversampling the Minority Class 185

9.5.4 Example: Classification of Forest Cover Type Data 186

9.6 Learning with Cost 190

9.7 Exercises 191

References 192

10 Assessing Classifier Performance 195

10.1 Classification Error and Other Measures of Predictive Power 195

10.2 Receiver Operating Characteristic (ROC) and Other Curves 196

10.2.1 Empirical ROC curve 196

10.2.2 Other Performance Measures 198

10.2.3 Optimal Operating Point 198

10.2.4 Area Under Curve 200

10.2.5 Smooth ROC Curves 200

10.2.6 Confidence Bounds for ROC Curves 205

10.3 Testing Equivalence of Two Classification Models 210

10.4 Comparing Several Classifiers 215

$10.5 \quad$ Exercises 217

References 218

11 Linear and Quadratic Discriminant Analysis, Logistic Regression, and Partial Least Squares Regression 221

11.1 Discriminant Analysis 221

11.1.1 Estimating the Covariance Matrix 223

11.1.2 Verifying Discriminant Analysis Assumptions 225

11.1.3 Applying LDA When LDA Assumptions Are Invalid 226

11.1.4 Numerical Implementation 228

11.1.5 Regularized Discriminant Analysis 228

11.1.6 LDA for Variable Transformation 229

11.2 Logistic Regression 231

11.2.1 Binomial Logistic Regression: Theory and Numerical Implementation 231

11.2.2 Properties of the Binomial Model 233

11.2.3 Verifying Model Assumptions 233

11.2.4 Logistic Regression with Multiple Classes 234

11.3 Classification by Linear Regression 235

$11.4 \quad 236$

11.5 Example: Linear Models for MAGIC Telescope Data 239

11.6 Choosing a Linear Classifier for Your Analysis 247

11.7 Exercises 247

References 248

$12 \quad$ Neural Networks 251

12.1 Perceptrons 251

12.2 The Feed-Forward Neural Network 254

12.3 Backpropagation 256 
12.4 Bayes Neural Networks 260

12.5 Genetic Algorithms 262

12.6 Exercises 263

References 263

13 Local Learning and Kernel Expansion 265

13.1 From Input Variables to the Feature Space 266

13.1.1 Kernel Regression 269

13.2 Regularization 270

13.2.1 Kernel Ridge Regression 274

13.3 Making and Choosing Kernels 278

13.4 Radial Basis Functions 279

13.4.1 Example: RBF Classification for the MAGIC Telescope Data 280

13.5 Support Vector Machines (SVM) 283

13.5.1 SVM with Weighted Data 286

13.5.2 SVM with Probabilistic Outputs 288

13.5.3 Numerical Implementation 288

13.5.4 Multiclass Extensions 293

13.6 Empirical Local Methods 293

13.6.1 Classification by Probability Density Estimation 294

13.6.2 Locally Weighted Regression 295

13.6.3 Nearest Neighbors and Fuzzy Rules 298

13.7 Kernel Methods: The Good, the Bad and the Curse of Dimensionality 302

$13.8 \quad$ Exercises 303

References 304

14 Decision Trees 307

14.1 Growing Trees 308

14.2 Predicting by Decision Trees 312

14.3 Stopping Rules 312

14.4 Pruning Trees 313

14.4.1 Example: Pruning a Classification Tree 317

14.5 Trees for Multiple Classes 319

14.6 So Splits on Categorical Variables 320

14.7 Surrogate Splits 321

14.8 Missing Values 323

14.9 Variable importance 324

14.10 Why Are Decision Trees Good (or Bad)? 327

14.11 Exercises 328

References 329

$15 \quad$ Ensemble Learning 331

15.1 Boosting 332

15.1.1 Early Boosting 332

15.1.2 AdaBoost for Two Classes 333 
15.1.3 Minimizing Convex Loss by Stagewise Additive Modeling 336

15.1.4 Maximizing the Minimal Margin 343

15.1.5 Nonconvex Loss and Robust Boosting 351

15.1.6 Boosting for Multiple Classes 357

15.2 Diversifying the Weak Learner: Bagging, Random Subspace and Random Forest 358

15.2.1 Measures of Diversity 359

15.2.2 Bagging and Random Forest 361

15.2.3 Random Subspace 363

15.2.4 Example: $K / \pi$ Separation for BaBar PID 364

15.3 Choosing an Ensemble for Your Analysis 365

15.4 Exercises 367

References 367

16 Reducing Multiclass to Binary 371

16.1 Encoding 372

16.2 Decoding 375

16.3 Summary: Choosing the Right Design 378

References 379

17 How to Choose the Right Classifier for Your Analysis and Apply It Correctly 381

17.1 Predictive Performance and Interpretability 381

17.2 Matching Classifiers and Variables 382

17.3 Using Classifier Predictions 382

17.4 Optimizing Accuracy 383

17.5 CPU and Memory Requirements 383

18 Methods for Variable Ranking and Selection 385

18.1 Definitions 386

18.1.1 Variable Ranking and Selection 386

18.1.2 Strong and Weak Relevance 386

18.2 Variable Ranking 389

18.2.1 Filters: Correlation and Mutual Information 390

18.2.2 Wrappers: Sequential Forward Selection (SFS), Sequential Backward Elimination (SBE), and Feature-based Sensitivity of Posterior Probabilities (FSPP) 394

18.2.3 Embedded Methods: Estimation of Variable Importance by Decision Trees, Neural Networks, Nearest Neighbors, and Linear Models 400

18.3 Variable Selection 401

18.3.1 Optimal-Set Search Strategies 401

18.3.2 Multiple Testing: Backward Elimination by Change in Margin (BECM) 403

18.3.3 Estimation of the Reference Distribution by Permutations: Artificial Contrasts with Ensembles (ACE) Algorithm 410

18.4 Exercises 413 
References 414

19 Bump Hunting in Multivariate Data 417

19.1 Voronoi Tessellation and SLEUTH Algorithm 418

19.2 Identifying Box Regions by PRIM and Other Algorithms 420

19.3 Bump Hunting Through Supervised Learning 422

References 423

20 Software Packages for Machine Learning 425

20.1 Tools Developed in HEP 425

20.2 R 426

20.3 MATLAB 427

20.4 Tools for Java and Python 428

20.5 What Software Tool Is Right for You? 429

References 430

Appendix A: Optimization Algorithms 431

A.1 Line Search 431

A.2 Linear Programming (LP) 432

Index 435 